\title{
A sharpened form of the inverse function theorem
}

Dedicated to Yuri Kozitsky on the occasion of his 70th birthday

\begin{abstract}
In this note we establish an advanced version of the inverse function theorem and study some local geometrical properties like starlikeness and hyperbolic convexity of the inverse function under natural restrictions on the numerical range of the underlying mapping.
\end{abstract}

\section{Introduction}

Let $D_{1}$ and $D_{2}$ be domains in the complex plane $\mathbb{C}$. By $\operatorname{Hol}\left(D_{1}, D_{2}\right)$ we denote the set of holomorphic mappings of $D_{1}$ into $D_{2}$. If $D$ is a domain in $\mathbb{C}$, then the set $\operatorname{Hol}(D):=\operatorname{Hol}(D, D)$ forms a semigroup with composition being the semigroup operation.

For $r>0$, we denote $\Delta_{r}:=\{z \in \mathbb{C}:|z|<r\}$ and $\Delta:=\Delta_{1}$.

The classical inverse function theorem states the following:

Theorem 1.1. Let function $\Phi$ be holomorphic in a neighborhood of the origin with $\Phi(0)=0$ and $\Phi^{\prime}(0) \neq 0$. Then there exist positive numbers $r$ and $\rho$ such that for all $z \in \Delta_{\rho}$ equation

$$
\Phi(w)=z
$$

2010 Mathematics Subject Classification. Primary 30D05; Secondary 47J07, 30C45.

Key words and phrases. Inverse function theorem, nonlinear resolvent, holomorphic function. 
has a unique solution $w=\Phi^{-1}(z)$ in the disk $\Delta_{r}$ and such that $\Phi^{-1} \in$ $\operatorname{Hol}\left(\Delta_{\rho}, \Delta_{r}\right)$.

Assuming that $\Phi$ is holomorphic in the open unit disk $\Delta$, the numbers $\rho$ and $r$ are often called the Bloch radii $[6,7]$. So, the natural problem is:

- To obtain qualified estimates for the Bloch radii for all functions $\Phi \in$ $\operatorname{Hol}(\Delta, \mathbb{C})$.

Standard methods of complex analysis for the Bloch radii problem include, for instance, employment of the maximum modulus of $|\Phi(z)|$ in the unit disk. Also, one can estimate the radius of convergence in the familiar Bürmann-Lagrange theorem. The last approach involves the consideration of the minimum of $\left|\frac{\Phi(z)}{z}\right|$ on some subsets of $\Delta$ or on the whole unit disk (see, for example, $[1,13]$ ).

In this connection, we observe that if a function $\Phi \in \operatorname{Hol}(\Delta, \mathbb{C}), \Phi(0)=0$, satisfies the condition

$$
\operatorname{Re}[\Phi(z) \bar{z}] \geq|z|^{2}, \quad z \in \Delta
$$

then by the argument principle, $\Phi$ covers the open unit disk $\Delta$ and for every $z \in \Delta$ there is a unique solution $w$ of the equation $\Phi(w)=z$.

In fact, this observation links to a notion, which comes from operator theory and nonlinear analysis. Namely, for a mapping $\Phi$, the numerical range of $\Phi$ is the set $N R(\Phi)$, given by

$$
N R(\Phi):=\{\zeta \in \mathbb{C}: \zeta=\Phi(z) \bar{z}, \quad z \in \Delta\} .
$$

Recall that traditionally one-sided estimates mean that the numerical range $N R(\Phi)$ of a mapping $\Phi$ lies in the left (right) half-plane.

Our goal in this paper is to study the following problem:

- Whether the Bloch radii can be given by using one-sided estimates in the spirit of Krasnoselskii?

Due to the maximal principle, for a function $\Phi$ satisfying $\Phi(0)=0$ this can be written in the form

$$
\pm \operatorname{Re} \frac{\Phi(z)}{z} \geq 0, \quad z \in \Delta .
$$

In other words, the problem above is the using of conditions of type (1.2) to get an appropriate estimates for the Bloch radii.

Note in passing that classical results of Marx [8] and Strohhäcker [12] state that any normalized convex function $\Phi$ satisfies $\operatorname{Re} \frac{\Phi(z)}{z}>\frac{1}{2}$. In the converse direction, it was shown by Noshiro [10] that if $\stackrel{z}{\Phi}(0)=0$ and $\operatorname{Re} \frac{\Phi(z)}{z} \geq 0$, then the function $\Phi$ is univalent and starlike in the disk $\Delta_{r}$ with $r=\sqrt{2}-1$. Obviously, by the Koebe Quarter Theorem one immediately obtains an estimate for the second Bloch radius $\rho$, namely, $\rho=(\sqrt{2}-1) / 4$. We show below that the inverse function $\Phi^{-1}$ is starlike in the disk $\Delta_{\rho}$. 
Another relevant question which arises in this connection is:

- Which conditions provide the equality of the numbers $\rho$ and $r$ ? In other words, does there exist a disk centered at the origin invariant for $\Phi^{-1}$ ? If yes, what is the radius of the maximal invariant disk?

As soon as we know the last radius, we can find the radius of the disk centered at the origin such that $\Phi^{-1}$ is a starlike of order $\frac{1}{2}$ self-mapping of this disk.

We should emphasize that, in fact, we study the above problems under a weaker restriction that $N R(\Phi)$ lies in an arbitrary half-plane, which is not necessarily vertical. Our results (see Section 3), in particular, imply the following conclusions.

Assertion 1.2. Let $\Phi \in \operatorname{Hol}(\Delta, \mathbb{C})$ satisfy $\left|\Phi^{\prime}(0)\right|>1$ and $\operatorname{Re} \frac{\Phi(w)}{\Phi^{\prime}(0) w}>0$. Then $\Phi^{-1} \in \operatorname{Hol}\left(\Delta_{R_{1}}, \Delta_{\sqrt{2}-1}\right)$, where $R_{1}=(3-2 \sqrt{2})\left|\Phi^{\prime}(0)\right|$. Furthermore, the inverse function $\Phi^{-1}$ preserves the disk $\Delta_{R}$ invariant, where

$$
R= \begin{cases}\frac{\left|\Phi^{\prime}(0)\right|-1}{\left|\Phi^{\prime}(0)\right|+1}, & \text { if }\left|\Phi^{\prime}(0)\right|<\sqrt{2}+1, \\ (3-2 \sqrt{2})\left|\Phi^{\prime}(0)\right|, & \text { if }\left|\Phi^{\prime}(0)\right| \geq \sqrt{2}+1 .\end{cases}
$$

Assertion 1.3. Let $\Phi \in \operatorname{Hol}(\Delta, \mathbb{C})$ satisfy $\operatorname{Re} \Phi^{\prime}(0)>1$ and $\operatorname{Re} \frac{\Phi(w)}{w}>0$. Then $\Phi^{-1} \in \operatorname{Hol}\left(\Delta_{R_{1}}, \Delta_{\sqrt{2}-1}\right)$, where $R_{1}=(3-2 \sqrt{2}) \operatorname{Re} \Phi^{\prime}(0)$. Furthermore, the inverse function $\Phi^{-1}$ preserves the disk $\Delta_{R}$ invariant, where

$$
R= \begin{cases}\frac{\operatorname{Re} \Phi^{\prime}(0)-1}{\operatorname{Re} \Phi^{\prime}(0)+1}, & \text { if } \operatorname{Re} \Phi^{\prime}(0)<\sqrt{2}+1, \\ (3-2 \sqrt{2}) \operatorname{Re} \Phi^{\prime}(0), & \text { if } \operatorname{Re} \Phi^{\prime}(0) \geq \sqrt{2}+1 .\end{cases}
$$

Assertion 1.4. Let $\Phi \in \operatorname{Hol}(\Delta, \mathbb{C})$ satisfy $\operatorname{Re} \Phi^{\prime}(0)>1$ and $\operatorname{Re} \frac{\Phi(w)}{w}>$ $\frac{1}{2} \operatorname{Re} \Phi^{\prime}(0)$. Then $\Phi^{-1} \in \operatorname{Hol}\left(\Delta_{R_{1}}, \Delta\right)$, where $R_{1}=\frac{1}{2} \operatorname{Re} \Phi^{\prime}(0)$. Furthermore, the inverse function $\Phi^{-1}$ preserves the disk $\Delta_{R}$ invariant, where

$$
R= \begin{cases}\frac{1}{2} \operatorname{Re} \Phi^{\prime}(0), & \text { if } \operatorname{Re} \Phi^{\prime}(0) \geq 2, \\ \operatorname{Re} \Phi^{\prime}(0)-1, & \text { if } \operatorname{Re} \Phi^{\prime}(0)<2 .\end{cases}
$$

\section{Preliminary notions and auxiliary results}

We start this section with the following definitions.

Definition 2.1. Let $D$ be a domain in $\mathbb{C}$ and let $h \in \operatorname{Hol}(D, \mathbb{C})$. One says that $h$ satisfies the range condition on $D$ if for each $\lambda \geq 0$ the following condition holds $(I+\lambda h)(D) \supset D$ and the equation

$$
w+\lambda h(w)=z
$$

has a unique solution

$$
w=\mathcal{J}_{\lambda}(z)\left(=(I+\lambda h)^{-1}(z)\right)
$$


holomorphic in $D$.

Usually the family $\left\{\mathcal{J}_{\lambda}\right\}_{\lambda \geq 0} \in \operatorname{Hol}(D)$ is called the resolvent family of $h$ on $D$.

Definition 2.2. Let $D=\Delta_{r}$ be the open disk of radius $r>0$ centered at the origin. A mapping $h \in \operatorname{Hol}(D, \mathbb{C})$ is said to be holomorphically accretive if there exists $\varepsilon>0$ such that

$$
\operatorname{Re} h(z) \bar{z} \geq 0
$$

for all $z$ satisfying $r-\varepsilon<|z|<r$.

It can be easily seen that if $h(0)=0$, then $h$ is holomorphically accretive if and only if its numerical range $N R(h)$ lies in the right half-plane. More detailed study of the numerical range of holomorphic mappings and its applications to different problems of analysis can be found in the recent book [3]. For our purpose we need the following fact.

Proposition 2.3. A mapping $h \in \operatorname{Hol}\left(\Delta_{r}, \mathbb{C}\right)$ is holomorphically accretive on $\Delta_{r}$ if and only if it satisfies the range condition on $\Delta_{r}$.

We now recall the notion of hyperbolically convex functions that has been studied by many authors; see, for example, [9].

Definition 2.4. A subdomain $D \subset \Delta_{r}$ is said to be a hyperbolically convex domain if for every pair of points $w_{1}, w_{2} \in D$, the hyperbolic geodesic segment joining $w_{1}$ and $w_{2}$ in $\Delta_{r}$ lies entirely in $D$.

Concerning geometric properties of the mappings $\mathcal{J}_{\lambda}$ in the case of the open unit disk $\Delta$, the following assertion was recently proved in [4].

Proposition 2.5. Let $h \in \operatorname{Hol}(\Delta, \mathbb{C})$ with $h(0)=0$ satisfy the range condition and let the family $\left\{\mathcal{J}_{\lambda}\right\}_{\lambda>0}$ be defined by (2.2). Then for each $\lambda>0$,

$$
\operatorname{Re} \mathcal{J}_{\lambda}^{\prime}(z)>0, \quad z \in \Delta,
$$

and $\mathcal{J}_{\lambda}(\Delta)$ is a hyperbolically convex domain. Consequently, the function $\mathcal{J}_{\lambda}$ is starlike of order $\frac{1}{2}$. If, in addition, $h^{\prime}(0)=\beta>0$, then

$$
\operatorname{Re} \frac{\mathcal{J}_{\lambda}(z)}{z}>\frac{1}{2(1+\beta \lambda)}, \quad z \in \Delta .
$$

We proceed with some technical lemmata that will be used in the proof of our main results.

Lemma 2.6. Let function $\phi$ be defined by

$$
\phi(\theta, S, t, r)=\frac{\cos \theta}{t}+\frac{2 S r^{2}}{t\left(1-r^{2}\right)}-\frac{2 S r}{|t|\left(1-r^{2}\right)}-1
$$

on the domain $\left\{\theta \in\left(-\frac{\pi}{2}, \frac{\pi}{2}\right) \cup\left(\frac{\pi}{2}, \frac{3 \pi}{2}\right), S>0, t \in \mathbb{R} \backslash\{0\}, r \in(0,1)\right\}$. Assume that one of the following conditions holds: 
(a) $\theta \in\left(-\frac{\pi}{2}, \frac{\pi}{2}\right), S<\cos \theta, t \in(0, \cos \theta-S]$;

(b) $\theta \in\left(-\frac{\pi}{2}, \frac{\pi}{2}\right), t \in(0, \cos \theta), r \leq \frac{\cos \theta-t}{2 S-(\cos \theta-t)}$;

(c) $\theta \in\left(\frac{\pi}{2}, \frac{3 \pi}{2}\right), t \in(\cos \theta, 0), r \leq \frac{t-\cos \theta}{2 S+t-\cos \theta}$.

Then

$$
\phi(\theta, S, t, r) \geq 0
$$

Proof. If $\theta \in\left(-\frac{\pi}{2}, \frac{\pi}{2}\right)$ and $t>0$, then inequality (2.5) is equivalent to

$$
\frac{\cos \theta}{t}-1-\frac{2 S r}{t(1+r)} \geq 0
$$

which coincides with $(1+r)(\cos \theta-t) \geq 2 r S$. The last inequality obviously holds for all $r \in(0,1)$ in case (a). So, we proceed with $t>\cos \theta-S$. Then (2.6) is equivalent to $r \leq \frac{\cos \theta-t}{2 S-(\cos \theta-t)}$. Thus (2.5) holds in case (b).

Let now consider the case $\theta \in\left(\frac{\pi}{2}, \frac{3 \pi}{2}\right)$ and $t<0$. Similarly to the above, condition (2.5) is equivalent to the inequality $\frac{\cos \theta}{t}-1+\frac{2 S r}{t(1-r)} \geq 0$. This implies that (2.5) holds in case (c) too.

Lemma 2.7. Let function $u$ be defined by

$$
u(\theta, S, t)= \begin{cases}t, & \text { as } \quad S<\cos \theta, 0<t \leq \cos \theta-S, \\ \frac{(\cos \theta-t) t}{2 S+t-\cos \theta}, & \text { otherwise }\end{cases}
$$

on the domain $\left\{\theta \in\left(-\frac{\pi}{2}, \frac{\pi}{2}\right), S>0, t \in(0, \cos \theta)\right\}$. Then for a fixed $\theta \in$ $\left(-\frac{\pi}{2}, \frac{\pi}{2}\right)$ and $S>0$,

$$
\max _{t \in(0, \cos \theta)} u(\theta, S, t)= \begin{cases}\cos \theta-S, & \text { as } S<\frac{2 \cos \theta}{3}, \\ 4 S-\cos \theta-2 \sqrt{(2 S-\cos \theta) 2 S}, & \text { as } S \geq \frac{2 \cos \theta}{3} .\end{cases}
$$

Proof. First we note that $u$ is a continuous function and that the function $\frac{(\cos \theta-t) t}{2 S+t-\cos \theta}$ with $S \leq \frac{\cos \theta}{2}$ is decreasing. For the case $S>\frac{\cos \theta}{2}$, the last function is increasing as $t<t_{0}:=\sqrt{(2 S-\cos \theta) 2 S}-2 S+\cos \theta$ and is decreasing otherwise.

We now use these facts to analyse the behavior of $u$. If $S \leq \frac{\cos \theta}{2}$, then $u(\theta, S, \cdot)$ is increasing as $t<t_{1}:=\cos \theta-S$ and decreasing as $t>t_{1}$. Therefore, its maximum is $u\left(\theta, S, t_{1}\right)$.

If $S \in\left(\frac{\cos \theta}{2}, \frac{2 \cos \theta}{3}\right)$, then $t_{0}<t_{1}$. Therefore, the same conclusion holds in this case.

If $S \in\left[\frac{2 \cos \theta}{3}, \cos \theta\right)$, then $t_{1} \leq t_{0}$, and hence $\max u(\theta, S, t)=u\left(\theta, S, t_{0}\right)$. Obviously, the last relation holds whenever $S \geq \cos \theta$. Computation of the values $u\left(\theta, S, t_{1}\right)$ and $u\left(\theta, S, t_{0}\right)$ completes the proof.

Lemma 2.8. Let function $v$ be defined by

$$
v(\theta, S, t)=\frac{(t-\cos \theta)|t|}{2 S+t-\cos \theta}
$$


on the domain $\left\{\theta \in\left(\frac{\pi}{2}, \frac{3 \pi}{2}\right), S>0, t \in(\cos \theta, 0)\right\}$. Then for fixed $\theta \in$ $\left(\frac{\pi}{2}, \frac{3 \pi}{2}\right)$ and $S>0$,

$$
\max _{t \in(\cos \theta, 0)} v(\theta, S, t)=4 S-\cos \theta-2 \sqrt{2 S(2 S-\cos \theta)} .
$$

Proof. Since the function $v(\theta, S, \cdot)$ attains its maximum at the point $t_{0}=$ $\sqrt{(2 S-\cos \theta) 2 S}-2 S+\cos \theta \in(\cos \theta, 0)$, the assertion follows.

\section{Main results}

Recall the main problem studied in this paper. Let $\Phi \in \operatorname{Hol}(\Delta, \mathbb{C})$ satisfy $\Phi(0)=0$. We are interested in the study of the inverse function $\Phi^{-1}$, in particular, in its domain and geometric properties. Therefore, it is natural to assume that $\alpha:=\Phi^{\prime}(0) \neq 0$; then the inverse function exists in a neighborhood of zero. Our additional assumption is that the function $\frac{\Phi(w)}{\alpha w}$ takes values in some not horizontal half-plane.

Our main result is the following assertion.

Theorem 3.1. Let $\Phi \in \operatorname{Hol}(\Delta, \mathbb{C})$ satisfy $\Phi(0)=0$ and $\alpha:=\Phi^{\prime}(0) \neq 0$. Assume also that for some positive $S$ and some $\theta \in\left(-\frac{\pi}{2}, \frac{\pi}{2}\right) \cup\left(\frac{\pi}{2}, \frac{3 \pi}{2}\right)$, the following inequality holds:

$$
\operatorname{Re} e^{i \theta} \frac{\Phi(w)}{\alpha w}>\cos \theta-S
$$

For $t \in \mathbb{R}$ such that $t \cos \theta>0$ and $|t|<|\cos \theta|$, denote

$$
r(\theta, S, t)=\left\{\begin{array}{l}
1, \quad \text { as } \theta \in\left(-\frac{\pi}{2}, \frac{\pi}{2}\right), t \in(0, \cos \theta-S], \\
\frac{\cos \theta-t}{2 S+t-\cos \theta}, \text { as } \theta \in\left(-\frac{\pi}{2}, \frac{\pi}{2}\right), t \in(\cos \theta-S, \cos \theta), t>0, \\
\frac{t-\cos \theta}{2 S+t-\cos \theta}, \text { as } \theta \in\left(\frac{\pi}{2}, \frac{3 \pi}{2}\right), t \in(\cos \theta, 0) .
\end{array}\right.
$$

Then for any $t$ the inverse function $\Phi^{-1}$ is holomorphic in the disk $\Delta_{\rho}$ with $\rho=r(\theta, S, t)|t \alpha|$ and maps $\Delta_{\rho}$ into $\Delta_{r}$ with $r=r(\theta, S, t)$.

Proof. Under the above assumptions, one can write $\Phi(w)=w q(w)$ with $q(0)=\alpha$ and $\operatorname{Re} e^{i \theta \frac{q(w)}{\alpha}}>\cos \theta-S$. Then the function $q_{1}$ defined by

$$
q_{1}(w):=\frac{1}{S}\left[e^{i \theta} \frac{q(w)}{\alpha}-\cos \theta+S\right]
$$

is of the Carathéodory class and hence satisfies

$$
\left|q_{1}(w)-\frac{1+|w|^{2}}{1-|w|^{2}}\right| \leq \frac{2|w|}{1-|w|^{2}}
$$

(see, for example, [5, Remark 2.1.4]). Denote $r:=|w|$. The last inequality is equivalent to the following one:

$$
\left|\frac{e^{i \theta} q(w)}{t \alpha}-\frac{\cos \theta}{t}-\frac{2 S r^{2}}{t\left(1-r^{2}\right)}\right| \leq \frac{2 S r}{|t|\left(1-r^{2}\right)},
$$


where $t$ is an arbitrary nonzero real number. In turn, this implies

$$
\operatorname{Re}\left[\frac{e^{i \theta} q(w)}{t \alpha}-1\right] \geq \frac{\cos \theta}{t}+\frac{2 S r^{2}}{t\left(1-r^{2}\right)}-\frac{2 S r}{|t|\left(1-r^{2}\right)}-1 .
$$

By Lemma 2.6, the right-hand side of this inequality is positive if one of the cases (a), (b) or (c) of that lemma holds. Consequently, Re $\left[\frac{e^{i \theta} q(w)}{t \alpha}-1\right]$ $\geq 0$ whenever $|w|<r(\theta, S, t)$, where the function $r(\theta, S, t)$ is defined by (3.2).

Then the function $Q$, defined by $Q(w):=\frac{e^{i \theta} q(r(\theta, S, t) w)}{t \alpha}-1$, has a positive real part in the unit disk. Thus, by Definition 2.2, the function $w Q(w)$ is a holomorphically accretive mapping in $\Delta$, and consequently, by Lemma 2.3, satisfies the range condition (see Definition 2.1). Therefore, for any positive $\lambda$ and for any $\hat{z} \in \Delta$, there is a solution $\hat{w}=w_{\lambda}(\hat{z})$ of the functional equation

$$
\hat{w}+\lambda \hat{w} Q(\hat{w})=\hat{z},
$$

which is holomorphic with respect to $\hat{z} \in \Delta$ and satisfies $\left|w_{\lambda}(\hat{z})\right|<1$. Obviously, for $\lambda=1$ the last equation gets the form

$$
\Phi(r(\theta, S, t) \hat{w})=r(\theta, S, t) t \alpha \hat{z} .
$$

Denoting $w=r(\theta, S, t) \hat{w}$ and $z=r(\theta, S, t) t \alpha \hat{z}$, we complete the proof.

Despite its generality, it is not convenient to apply Theorem 3.1 directly because its conclusion contains an arbitrary parameter $t$. So, our next purpose is to obtain readable results without additional parameters.

Corollary 3.2. Let $\Phi \in \operatorname{Hol}(\Delta, \mathbb{C})$ satisfy $\Phi(0)=0$ and $\alpha:=\Phi^{\prime}(0) \neq 0$. Assume that inequality (3.1) holds for some $S>0$ and $\theta \in\left(-\frac{\pi}{2}, \frac{\pi}{2}\right)$. The inverse function $\Phi^{-1}$ is holomorphic in the disk $\Delta_{R_{1}(\theta, S)}$, where

$$
R_{1}(\theta, S)= \begin{cases}|\alpha|(\cos \theta-S), & \text { as } S<\frac{2 \cos \theta}{3}, \\ |\alpha|(4 S-\cos \theta-2 \sqrt{(2 S-\cos \theta) 2 S}), & \text { as } S \geq \frac{2 \cos \theta}{3},\end{cases}
$$

and maps it onto a hyperbolically convex subdomain of the disk $\Delta_{R_{2}(\theta, S)}$, where

$$
R_{2}(\theta, S)= \begin{cases}1, & \text { as } S<\frac{2 \cos \theta}{3}, \\ \frac{2 S}{\sqrt{(2 S-\cos \theta) 2 S}}-1, & \text { as } S \geq \frac{2 \cos \theta}{3} .\end{cases}
$$

Proof. Indeed, the statement of Theorem 3.1 involves the real parameter $t$. Therefore, we can maximize $r(\theta, S, t)|t \alpha|$ as the function of variable $t \in$ $(0, \cos \theta)$. This was done in Lemma 2.7. The hyperbolic convexity follows from Theorem 2.5.

Similarly, using Lemma 2.8 instead of Lemma 2.7, we get the following assertion. 
Corollary 3.3. Let $\Phi \in \operatorname{Hol}(\Delta, \mathbb{C})$ satisfy $\Phi(0)=0$ and $\alpha:=\Phi^{\prime}(0) \neq 0$. Assume that inequality (3.1) holds for some $S>0$ and $\theta \in\left(\frac{\pi}{2}, \frac{3 \pi}{2}\right)$. The inverse function $\Phi^{-1}$ is holomorphic in the disk $\Delta_{R_{1}(\theta, S)}$, where

$$
R_{1}(\theta, S)=|\alpha|(4 S-\cos \theta-2 \sqrt{(2 S-\cos \theta) 2 S})
$$

and maps it onto a hyperbolically convex subdomain of the disk $\Delta_{R_{2}(\theta, S)}$, where

$$
R_{2}(\theta, S)=1-\frac{2 S}{\sqrt{(2 S-\cos \theta) 2 S}} .
$$

Corollary 3.4. If in the hypotheses of Theorem 3.1, either $\theta=0$ or $\theta=\pi$, then the inverse function satisfies the following inequality:

$$
\operatorname{Re} \frac{\alpha \Phi^{-1}(z) \cos \theta}{z}>\frac{1}{2}, \quad|z|<R_{1}(\theta, S),
$$

where the function $R_{1}(\theta, S)$ is defined in Corollaries 3.2-3.3.

Proof. Let us return to equation (3.4) and consider the function $h(\hat{w})=$ $\hat{w} Q(\hat{w})$. If either $\theta=0$ or $\theta=\pi$, then $\beta:=h^{\prime}(0)=\frac{\cos \theta}{t}-1>0$. It follows from Theorem 2.5 that the function $\hat{w}=w_{1}(\hat{z})$ satisfies

$\operatorname{Re} \frac{\hat{w}}{\tilde{z}}>\frac{t}{2 \cos \theta}, \hat{z} \in \Delta$. This is equivalent to the inequality $\operatorname{Re} \frac{\alpha \Phi^{-1}(z) \cos \theta}{z}>$ $\frac{1}{2}$ in the disk $|z|<r(\theta, S, t)|t \alpha|$. Now Corollaries 3.2-3.3 imply the conclusion.

Concerning invariant disks, it is clear that they exist whenever $|\alpha|>1$. At the same time, the classical Theorem 1.1 does not answer how to estimate the size of such disks. Under the stronger condition $|\alpha \cos \theta|>1$, Theorem 3.1 enables us to do this. Indeed, the disks described in this theorem are invariant whenever $|t \alpha| \geq 1$ while $|t|<|\cos \theta|$. Furthermore, in order to find maximal invariant disks, we have, on the way similar to Lemmata 2.7-2.8, maximize the functions $u$ and $v$ with respect to $t,|t| \geq \frac{1}{|\alpha|}$. Repeating the above considerations, we get the following conclusion.

Corollary 3.5. Let $\Phi \in \operatorname{Hol}(\Delta, \mathbb{C})$ satisfy $\Phi(0)=0$ and $\alpha=\Phi^{\prime}(0)$. Assume that for some $\theta \in\left(-\frac{\pi}{2}, \frac{\pi}{2}\right) \cup\left(\frac{\pi}{2}, \frac{3 \pi}{2}\right)$ and $S>0$, the inequalities $|\alpha \cos \theta|>1$ and (3.1) hold. Then the inverse function $\Phi^{-1}$ preserves the disk $\Delta_{R(\theta, S)}$ invariant, where $R(\theta, S)$ is defined as follows. Denote $G=4 S-\cos \theta-$ $2 \sqrt{(2 S-\cos \theta) 2 S}, t_{0}=\sqrt{(2 S-\cos \theta) 2 S}-2 S+\cos \theta$ and $t_{1}=\cos \theta-S$. In the case $\theta \in\left(-\frac{\pi}{2}, \frac{\pi}{2}\right)$,

$$
R(\theta, S)= \begin{cases}|\alpha| t_{1}, & \text { if } S<\frac{2 \cos \theta}{3}, \frac{1}{|\alpha|} \leq t_{1}, \\ \frac{|\alpha| \cos \theta-1}{|\alpha|(2 S-\cos \theta)+1}, & \text { if either } S<\frac{2 \cos \theta}{3}, \frac{1}{|\alpha|}>t_{1}, \\ |\alpha| G, & \text { or } S \geq \frac{2 \cos \theta}{3}, \frac{1}{|\alpha|}>t_{0}, \\ |\alpha| G \geq \frac{2 \cos \theta}{3}, \frac{1}{|\alpha|} \leq t_{0} .\end{cases}
$$


In the case $\theta \in\left(\frac{\pi}{2}, \frac{3 \pi}{2}\right)$,

$$
R(\theta, S)= \begin{cases}|\alpha| G, & \text { if } \frac{1}{|\alpha|} \leq t_{0}, \\ \frac{1-|\alpha| \cos \theta}{|\alpha|(2 S-\cos \theta)+1}, & \text { if } \frac{1}{|\alpha|}>t_{0} .\end{cases}
$$

To complete the paper we mention that one can obtain Assertions 1.2-1.4 above, choosing appropriate values of the parameters $S$ and $\theta$ with $\cos \theta>0$ in the statements of this section. Similarly, one can explore the case where $\cos \theta<0$.

\section{References}

[1] Aizenberg, L. A., Yuzhakov, A. P., Integral Representations and Residues in Multidimensional Complex Analysis, Translations of mathematical monographs 58, AMS, Providence, R.I., 1983.

[2] Elin, M., Shoikhet, D., Linearization Models for Complex Dynamical Systems. Topics in Univalent Functions, Functional Equations and Semigroup Theory, Birkhäuser, Basel, 2010.

[3] Elin, M., Reich, S., Shoikhet, D., Numerical Range of Holomorphic Mappings and Applications, Birkhäuser, Basel, 2019.

[4] Elin, M., Shoikhet, D., Sugawa, T., Geometric properties of the nonlinear resolvent of holomorphic generators, J. Math. Anal. Appl. 483 (2020), Art. 123614.

[5] Graham, I., Kohr, G., Geometric Function Theory in One and Higher Dimensions, Marcel Dekker, Inc., NY-Basel, 2003.

[6] Harris, L. A., On the size of balls covered by analytic transformations, Monatshefte Math. 83 (1977), 9-23.

[7] Harris, L. A., Reich, S., Shoikhet, D., Dissipative holomorphic functions, Bloch radii, and the Schwarz Lemma, J. Analyse Math. 82 (2000), 221-232.

[8] Marx, A., Untersuchungen über schlichte Abbildungen, Math. Ann., 107 (1933), 4067.

[9] Mejía, D., Pommerenke, Ch., On hyperbolically convex functions, J. Geom. Anal. 10 (2000), 365-378.

[10] Noshiro, K., On the univalency of certain analytic functions, J. Fac. Sci., Hokkaido Imp. Univ. Ser. I Math. 2 (1934), 89-101.

[11] Shoikhet, D., Semigroups in Geometrical Function Theory, Kluwer Academic Publishers, Dordrecht, 2001.

[12] Strohhäcker, E., Beiträge zur Theorie der schlichten Funktionen, Math. Z., 37 (1933), 356-380.

[13] Whittaker, E. T., Watson, G. N., A Course of Modern Analysis, Cambridge University Press, 1996.

Mark Elin

ORT Braude College

P.O. Box 78, Karmiel 21982

Israel

e-mail: mark_elin@braude.ac.il
David Shoikhet

Holon Institute of Technology

52 Golomb Street, POB 305 Holon 5810201

Israel

e-mail: davidsho@it.ac.il

Received July 28, 2019 